Proceedings of the Edinburgh Mathematical Society (2003) 46, 465-488 (C)

DOI:10.1017/S0013091502000500 Printed in the United Kingdom

\title{
INTEGRAL FORMULAE FOR SPACELIKE HYPERSURFACES IN CONFORMALLY STATIONARY SPACETIMES AND APPLICATIONS
}

\author{
LUIS J. ALÍAS ${ }^{1}$, ALDIR BRASIL JR ${ }^{2}$ AND A. GERVASIO COLARES ${ }^{2}$ \\ ${ }^{1}$ Departamento de Matemáticas, Universidad de Murcia, \\ E-30100 Espinardo, Murcia, Spain (ljalias@um.es) \\ ${ }^{2}$ Departamento de Matemática, Universidade Federal do Ceará, Campus do Pici, \\ 60455-760 Fortaleza-Ce, Brazil (aldir@mat.ufc.br; gcolares@mat.ufc.br)
}

(Received 13 May 2002)

\begin{abstract}
In this paper we develop general Minkowski-type formulae for compact spacelike hypersurfaces immersed into conformally stationary spacetimes, that is, Lorentzian manifolds admitting a timelike conformal field. We apply them to the study of the umbilicity of compact spacelike hypersurfaces in terms of their $r$-mean curvatures. We derive several uniqueness results, for instance, compact spacelike hypersurfaces are umbilical if either some of their $r$-mean curvatures are linearly related or one of them is constant.
\end{abstract}

Keywords: timelike conformal vector field; conformally stationary spacetime; spacelike hypersurface; $r$-mean curvatures; Newton transformations; Minkowski-type formulae

2000 Mathematics subject classification: Primary 53C42

Secondary 53B30; 53C50; 53Z05; 83C99

\section{Introduction}

Interest in the study of spacelike hypersurfaces in Lorentzian manifolds has increased very much in recent years, from both the physical and mathematical points of view. A basic question on this topic is the uniqueness of spacelike hypersurfaces with some natural geometric properties, like the vanishing or constancy of the mean curvature. A first relevant result in this direction was the proof of the Calabi-Bernstein conjecture for maximal hypersurfaces (that is, zero mean curvature spacelike hypersurfaces) in Lorentz-Minkowski space given by Cheng and Yau [12]. As for the case of de Sitter space, Goddard [17] conjectured that every complete spacelike hypersurface with constant mean curvature in de Sitter space should be totally umbilical. Although the conjecture turned out to be false in its original statement, it motivated a great deal of work of several authors trying to find a positive answer to the conjecture under appropriate additional hypotheses $[\mathbf{1}, \mathbf{2 5}, \mathbf{2 9}]$. For an account of the subject, see the review article by the second and third authors $[\mathbf{1 0}]$.

As for the case of more general Lorentzian ambient spaces, in a series of recent papers, the first author together with Romero and Sánchez [5-7] studied the uniqueness of space- 
like hypersurfaces with constant mean curvature in a wide class of Lorentzian manifolds, the so-called conformally stationary spacetimes. Since our work will be developed in such ambient spacetimes, we will describe here that class of Lorentzian manifolds, and its relation to other known spacetimes, such as generalized Robertson-Walker spacetimes. Throughout this paper, $\bar{M}$ will denote a conformally stationary spacetime, that is, $\bar{M}$ will be a manifold endowed with a Lorentzian metric tensor $\langle$,$\rangle , which will be equipped$ with a timelike conformal vector field $K \in \mathcal{X}(\bar{M})$. The fact that $K$ is conformal means that the Lie derivative of the Lorentzian metric $\langle$,$\rangle with respect to K$ satisfies

$$
\mathcal{L}_{K}\langle,\rangle=2 \phi\langle,\rangle
$$

for a certain smooth function $\phi \in \mathcal{C}^{\infty}(\bar{M})$. In particular, when $K$ is a Killing field $(\phi \equiv 0)$, then $\bar{M}$ is classically called a stationary spacetime. The reason for the terminology conformally stationary spacetime in our context is due to the fact that $\bar{M}$ endowed with the conformally related metric $\langle,\rangle^{*}=(1 /|K|)\langle$,$\rangle , where |K|=\sqrt{-\langle K, K\rangle}>0$, is in fact a stationary spacetime, since the timelike field $K$ is a Killing field for $\langle,\rangle^{*}[\mathbf{3 7}$, Lemma 2.1]. From a purely mathematical interest, stationary spacetimes have been recently studied by different authors in order to obtain geodesic completeness of Lorentzian manifolds $[\mathbf{2 1}, \mathbf{3 1}]$ as well as interesting classification results [32]. On the other hand, Sánchez [36] studied the geometry of stationary spacetimes from several points of view, some of them of physical interest.

In general relativity, symmetry is usually based on a local one-parameter group of isometries generated by a Killing or, more generally, a conformal vector field $K$. In fact, the main simplification for the search of exact solutions to the Einstein equation is to assume, a priori, the existence of such symmetries $[\mathbf{1 3}, \mathbf{1 5}]$. Even more useful has been the use of affine and affine conformal vector fields in obtaining new exact solutions [14]. Although in such approaches the causal character of the Killing or conformal vector field $K$ is not always presumed, it is natural to assume that $K$ is timelike. This is supported by very well-known examples of exact solutions. Moreover, when $K$ is timelike its integral curves become a privileged class of observers (in the sense of [35]) or test particles in spacetime.

The class of conformally stationary spacetimes includes the family of generalized Robertson-Walker spacetimes. By a generalized Robertson-Walker spacetime, we mean a Lorentzian warped product $\bar{M}=-I \times_{f} F^{n}$ with Riemannian fibre $F$ and warping function $f$. In that case, the timelike conformal field $K$ is given by $K(t, p)=f(t)(\partial / \partial t)_{(t, p)}$ and $\phi(t, p)=f^{\prime}(t)$. Moreover, by observing that a Lorentzian manifold which is globally conformal to a conformally stationary spacetime is itself a conformally stationary spacetime, it follows that conformally stationary spacetime also includes those Lorentzian manifolds which are globally conformal to generalized Robertson-Walker spacetimes as well as to stationary spacetimes. We refer the reader to $[\mathbf{1 9}]$ and $[\mathbf{3 3}]$ for some recent advances on the global structure of conformally stationary spacetimes. It is interesting to observe that, for a generalized Robertson-Walker spacetime, the conformal field $K$ is also closed, in the sense that its metrically equivalent 1-form is closed. As observed by Montiel $[\mathbf{2 5}]$, if $\bar{M}$ is a conformally stationary spacetime with a closed conformal field, then it 
is locally isometric to a generalized Robertson-Walker spacetime. For a global analogue of this assertion, under the assumption of timelike geodesic completeness, see [25, Proposition 2], where the author proves that it is isometric to an appropriate quotient of a generalized Robertson-Walker spacetime.

Under diverse hypotheses on the ambient space which come naturally either from a mathematical point of view (constant sectional curvature spacetimes and, more generally, Einstein spacetimes) or from a physical one (timelike convergence condition and, more generally, null convergence condition), Alías, Romero and Sánchez were able to prove that every compact spacelike hypersurface with constant mean curvature must be totally umbilical. In the case where the timelike conformal field is assumed to be closed, then the spacetime admits a foliation by totally umbilical spacelike hypersurfaces with constant mean curvature. In [26] Montiel classified the totally umbilical hypersurfaces with constant mean curvature of such spacetimes in terms of that foliation, under the hypothesis of the null convergence condition on the spacetime. He also obtained a uniqueness result for the case of spacelike hypersurfaces with constant scalar curvature.

The natural generalization of mean and scalar curvatures for an $n$-dimensional hypersurface is the $r$-mean curvatures $H_{r}$, for $r=1, \ldots, n$. In fact, $H_{1}$ is just the mean curvature and $H_{2}$ defines a geometric quantity which is related to the scalar curvature. For instance, if the ambient space has constant sectional curvature $\bar{c}$, then the scalar curvature of the hypersurface is given by $S=n(n-1)\left(\bar{c}-H_{2}\right)$ (for the details, see $\S 2$ ). Therefore, it is natural to think of extending those previous results to the case of higherorder $r$-mean curvatures.

In order to do that, we will develop here general Minkowski-type formulae for spacelike hypersurfaces immersed into conformally stationary spacetimes. The use of those kinds of integral formulae in the Lorentzian setting was started by Montiel in [25] for the case of spacelike hypersurfaces with constant mean curvature in de Sitter space, and it was continued by the first author together with Romero and Sánchez for the case of spacelike hypersurfaces with constant mean curvature in more general spacetimes (generalized Robertson-Walker spacetimes $[\mathbf{5}, \mathbf{6}]$ and, more generally, conformally stationary spacetimes $[\mathbf{7}])$. Observe that for the case of the mean curvature only the first and the second Minkowski formulae are needed. These two Minkowski formulae were obtained in [7] for the case of spacelike hypersurfaces in conformally stationary spacetimes, although they were not called Minkowski formulae.

Higher-order Minkowski formulae for hypersurfaces were first obtained by Hsiung [20] in Euclidean space, and by Bivens [9] in the Euclidean sphere and hyperbolic space. These were generalized by Alencar and the third author [3] by using the $(r+1)$-mean curvature linearized operator $L_{r}$ of the hypersurface.

In the Lorentzian setting, the first attempt to obtain higher-order Minkowski formulae was made by the first author together with Aledo and Romero in [2], where they developed the corresponding Minkowski formulae for spacelike hypersurfaces in de Sitter space, and applied them to characterize the totally umbilical round spheres as the only compact spacelike hypersurfaces with constant $r$-mean curvature in de Sitter space, under appropriate conditions. On the other hand, Montiel [26] gave another proof of 
the first and second Minkowski formulae for the case of spacelike hypersurfaces in conformally stationary spacetimes with closed conformal field. His proof uses the method of parallel hypersurfaces, which has a very nice geometric interpretation. However, that method is very difficult to carry out for successive higher-order Minkowski formulae, since it involves covariant derivatives of the Ricci tensor of the ambient space. In fact, for deriving the next Minkowski formula (the third one), Montiel needed to assume that the ambient space has constant sectional curvature.

In this paper we will develop another method which follows the ideas of Reilly [30]. In fact, we will obtain the correct integrands of our integral formulae as the divergences of vector fields, in the spirit of the method developed in [3], which uses the operator $L_{r}$ applied to the corresponding functions. Although more analytic than geometric, this method has the advantage of working for any higher-order mean curvature, allowing us to obtain general Minkowski-type formulae (Theorem 4.1 and Corollaries 4.2 and 4.3). Besides, we apply them to the study of the umbilicity of spacelike hypersurfaces with constant $r$-mean curvature, extending some previous results to the case of higher-order mean curvatures. For instance, we show (Theorem 5.2) that

in a conformally stationary spacetime of constant sectional curvature, the only compact spacelike hypersurfaces having $H_{r-1}$ and $H_{r}$ both constant, with $1 \leqslant r \leqslant n-1$, are the totally umbilical ones.

For the case of scalar curvature we extend [26, Theorem 8 ] to the case of general conformally stationary spacetimes (Theorem 5.3), and for higher-order $r$-mean curvatures we show the following (Theorem 5.10).

Let $M^{n}, n \geqslant 3$, be a compact spacelike hypersurface immersed into a conformally stationary-closed spacetime $\bar{M}$ with constant sectional curvature $\bar{c}$. Assume that Div $K$ does not vanish on $M$, where $K$ denotes the closed conformal timelike vector field on $\bar{M}$. If $M$ has constant $r$ th mean curvature $H_{r}$, $1 \leqslant r \leqslant n$, then $M$ is totally umbilical in $\bar{M}$. Besides, if $\bar{c} \leqslant 0$, then $M$ must be a leaf of the foliation $\mathcal{F}(K)$, and if $\bar{c}>0$, then $M$ is either a leaf of $\mathcal{F}(K)$ or a round umbilical sphere.

Here by conformally stationary-closed spacetime we mean a conformally stationary spacetime whose conformal timelike field is closed. The condition that Div $K$ does not vanish on the hypersurface assures the existence of an elliptic point, which is needed to apply Gärding inequalities (see Corollary 5.5 and a stronger version in Lemma 5.4). It is worth pointing out that such a condition has an interesting physical interpretation in terms of the average behaviour of the observers in the direction of $K$, as well as a geometric content in terms of the critical points of the metric along the integral curves of $K$ (see Remark 5.8). In particular, when $\bar{M}$ is a generalized Robertson-Walker spacetime, our result is stated as follows (Corollary 5.12).

Let $\bar{M}=-I \times_{f} F$ be a generalized Robertson-Walker spacetime (necessarily with compact Riemannian fibre $F$ ) with constant sectional curvature. Then the only compact spacelike hypersurfaces immersed into $\bar{M}$ with constant 
$r$-mean curvature on which $f^{\prime}$ does not vanish are the non-totally-geodesic spacelike slices $\{t\} \times F$, with $t \in I$ and $f^{\prime}(t) \neq 0$, except in the case where $\bar{M}$ is isometric to de Sitter spacetime in a neighbourhood of the hypersurface $M$, which must be a round umbilical hypersphere.

In $\S 6$ we give another application of our Minkowski-type formulae to the study of the umbilicity of spacelike hypersurfaces with a characteristic behaviour on their $r$-mean curvatures. Specifically, we obtain that the only compact spacelike hypersurfaces immersed into a conformally stationary-closed spacetime whose $r$-mean curvatures are linearly related are the totally umbilical ones, under the hypothesis that Div $K$ does not vanish on the hypersurface (Theorem 6.1).

\section{Preliminaries}

Let $\bar{M}^{n+1}$ be an $(n+1)$-dimensional $(n \geqslant 2)$ conformally stationary spacetime, endowed with a Lorentzian metric tensor $\langle$,$\rangle and with a timelike conformal vector field K \in$ $\mathcal{X}(\bar{M})$. Recall that the fact that $K$ is conformal means that the Lie derivative of the Lorentzian metric $\langle$,$\rangle with respect to K$ satisfies

$$
\mathcal{L}_{K}\langle,\rangle=2 \phi\langle,\rangle
$$

for a certain smooth function $\phi \in \mathcal{C}^{\infty}(\bar{M})$. In other words,

$$
\left\langle\bar{\nabla}_{V} K, W\right\rangle+\left\langle V, \bar{\nabla}_{W} K\right\rangle=2 \phi\langle V, W\rangle,
$$

for all vector fields $V, W \in \mathcal{X}(\bar{M})$, where $\bar{\nabla}$ stands for the Levi-Civita connection of $\bar{M}$.

A smooth immersion $\psi: M^{n} \rightarrow \bar{M}^{n+1}$ of an $n$-dimensional connected manifold $M$ is said to be a spacelike hypersurface if the induced metric via $\psi$ is a Riemannian metric on $M$, which, as usual, is also denoted by $\langle$,$\rangle . Since the timelike vector field K \in \mathcal{X}(\bar{M})$ is globally defined on $\bar{M}$, it determines a time-orientation on $\bar{M}$. Thus, for a given spacelike hypersurface $\psi: M \rightarrow \bar{M}$, there exists a unique timelike unit normal field $N$ globally defined on $M$ which is in the same time-orientation as $K$, so that

$$
\langle K, N\rangle \leqslant-|K|=-\sqrt{-\langle K, K\rangle}<0
$$

holds everywhere on $M$. If $\nabla$ denotes the Levi-Civita connection of $M$, then the Gauss and Weingarten formulae for the hypersurface in $\bar{M}$ are given, respectively, by

$$
\bar{\nabla}_{X} Y=\nabla_{X} Y-\langle A X, Y\rangle N
$$

and

$$
A(X)=-\bar{\nabla}_{X} N
$$

for all tangent vector fields $X, Y \in \mathcal{X}(M)$. Here $A: \mathcal{X}(M) \rightarrow \mathcal{X}(M)$ defines the shape operator of $M$ with respect to $N$. 
As is well known, the curvature tensor $R$ of the hypersurface $M$ is described in terms of $\bar{R}$, the curvature tensor of the ambient spacetime $\bar{M}$, and the shape operator of $M$ by the so-called Gauss equation, which can be written as

$$
R(X, Y) Z=(\bar{R}(X, Y) Z)^{\mathrm{T}}-\langle A X, Z\rangle A Y+\langle A Y, Z\rangle A X
$$

for all tangent vector fields $X, Y, Z \in \mathcal{X}(M)$, where $(\bar{R}(X, Y) Z)^{\mathrm{T}}$ denotes the tangential component of $\bar{R}(X, Y) Z$. Observe that our choice for the curvature tensor is the one in $[\mathbf{2 8}]$. On the other hand, the Codazzi equation of the hypersurface describes the normal component of $\bar{R}(X, Y) Z$ in terms of the derivative of the shape operator, and it is given by

$$
\langle\bar{R}(X, Y) Z, N\rangle=\left\langle\left(\nabla_{Y} A\right) X-\left(\nabla_{X} A\right) Y, Z\right\rangle,
$$

where $\nabla_{X} A$ denotes the covariant derivative of $A$ (we refer the reader to $[\mathbf{2 8}$, Chapter 4] for the details). In particular, when the ambient spacetime has constant sectional curvature, then $\bar{R}(X, Y) Z$ is tangent to $M$ for every $X, Y, Z \in \mathcal{X}(M)$, and (2.5) becomes

$$
\left(\nabla_{Y} A\right) X=\left(\nabla_{X} A\right) Y .
$$

Associated with the shape operator of $M$ there are $n$ algebraic invariants, which are the elementary symmetric functions $\sigma_{r}$ of its principal curvatures $\kappa_{1}, \ldots, \kappa_{n}$, given by

$$
\sigma_{r}\left(\kappa_{1}, \ldots, \kappa_{n}\right)=\sum_{i_{1}<\cdots<i_{r}} \kappa_{i_{1}} \ldots \kappa_{i_{r}}, \quad 1 \leqslant r \leqslant n .
$$

The $r$ th mean curvature $H_{r}$ of the spacelike hypersurface is then defined by

$$
\left(\begin{array}{l}
n \\
r
\end{array}\right) H_{r}=(-1)^{r} \sigma_{r}\left(\kappa_{1}, \ldots, \kappa_{n}\right)=\sigma_{r}\left(-\kappa_{1}, \ldots,-\kappa_{n}\right) .
$$

In particular, when $r=1$,

$$
H_{1}=-\frac{1}{n} \sum_{i=1}^{n} \kappa_{i}=-\frac{1}{n} \operatorname{trace}(A)=H
$$

is the mean curvature of $M$, which is the main extrinsic curvature of the hypersurface. The choice of the sign $(-1)^{r}$ in our definition of $H_{r}$ is motivated by the fact that in that case the mean curvature vector is given by $\vec{H}=H N$. Therefore, $H(p)>0$ at a point $p \in M$ if and only if $\vec{H}(p)$ is in the same time-orientation as $N(p)$, and hence as $K(p)$.

When $r=2, \mathrm{H}_{2}$ defines a geometric quantity which is related to the (intrinsic) scalar curvature of the hypersurface. Indeed, it follows from the Gauss equation (2.4) that the Ricci curvature of $M$ is given by

$$
\operatorname{Ric}(X, Y)=\overline{\operatorname{Ric}}(X, Y)+\langle\bar{R}(X, N) Y, N\rangle-\operatorname{trace}(A)\langle A X, Y\rangle+\langle A X, A Y\rangle,
$$

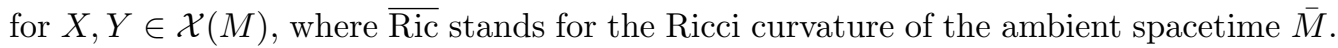
Therefore, the scalar curvature $S$ of the hypersurface $M$ is

$$
S=\operatorname{trace}(\operatorname{Ric})=\bar{S}+2 \overline{\operatorname{Ric}}(N, N)-n(n-1) H_{2} .
$$


For instance, if the ambient spacetime is Einstein with $\overline{\operatorname{Ric}}=\bar{C}\langle\rangle,, \bar{C}$ being a real constant, then $\bar{S}=(n+1) \bar{C}$ and $n(n-1) H_{2}=(n-1) \bar{C}-S$. In particular, when the ambient spacetime has constant sectional curvature $\bar{c}$ we obtain that

$$
S=n(n-1)\left(\bar{c}-H_{2}\right) .
$$

\section{The Newton transformations}

In this section we will introduce the corresponding Newton transformations

$$
T_{r}: \mathcal{X}(M) \rightarrow \mathcal{X}(M), \quad 0 \leqslant r \leqslant n,
$$

which arise from the shape operator $A$. These Newton transformations will be used in the next section to derive our integral formulae. According to our definition of the $r$ th mean curvatures, the Newton transformations are given by

$$
T_{r}=\left(\begin{array}{c}
n \\
r
\end{array}\right) H_{r} I+\left(\begin{array}{c}
n \\
r-1
\end{array}\right) H_{r-1} A+\cdots+\left(\begin{array}{c}
n \\
1
\end{array}\right) H_{1} A^{r-1}+A^{r},
$$

where $I$ denotes the identity in $\mathcal{X}(M)$, or, inductively,

$$
T_{0}=I \quad \text { and } \quad T_{r}=\left(\begin{array}{c}
n \\
r
\end{array}\right) H_{r} I+A \circ T_{r-1}
$$

Observe that the characteristic polynomial of $A$ can be written in terms of the $H_{r}$ as

$$
\operatorname{det}(t I-A)=\sum_{r=0}^{n}\left(\begin{array}{l}
n \\
r
\end{array}\right) H_{r} t^{n-r},
$$

where $H_{0}=1$. By the Cayley-Hamilton Theorem, this implies that $T_{n}=0$.

Let us remark that $T_{r}=(-1)^{r} \tilde{T}_{r}$, where $\tilde{T}_{r}$ is the $r$ th Newton transformation defined by Reilly in [30] for the case of hypersurfaces in a Riemannian space form (see also [34] for a more accessible modern treatment given by Rosenberg). The use of the Newton transformations for the case of spacelike hypersurfaces in Lorentzian spaces was introduced by the first author, jointly with Aledo and Romero, in [2], where they were applied to the study of compact spacelike hypersurfaces in de Sitter space with constant higher-order mean curvature.

Observe that the Newton transformations $T_{r}$ are all self-adjoint operators which commute with the shape operator $A$. Moreover, if $\left\{e_{1}, \ldots, e_{n}\right\}$ is an orthonormal frame on $T_{p} M$ which diagonalizes $A_{p}, A_{p}\left(e_{i}\right)=\kappa_{i}(p) e_{i}$, then

$$
\left(T_{r}\right)_{p}\left(e_{i}\right)=\lambda_{i, r}(p) e_{i}
$$

where

$$
\lambda_{i, r}=(-1)^{r} \sum_{i_{1}<\cdots<i_{r}, i_{j} \neq i} \kappa_{i_{1}} \ldots \kappa_{i_{r}}=\sum_{i_{1}<\cdots<i_{r}, i_{j} \neq i}\left(-\kappa_{i_{1}}\right) \cdots\left(-\kappa_{i_{r}}\right) .
$$


It follows from here that for each $r, 0 \leqslant r \leqslant n-1$,

$$
\operatorname{trace}\left(T_{r}\right)=c_{r} H_{r} \quad \text { and } \quad \operatorname{trace}\left(A \circ T_{r}\right)=-c_{r} H_{r+1},
$$

where

$$
c_{r}=(n-r)\left(\begin{array}{l}
n \\
r
\end{array}\right)=(r+1)\left(\begin{array}{c}
n \\
r+1
\end{array}\right) .
$$

The divergence of $T_{r}$ is defined by

$$
\operatorname{div} T_{r}=\operatorname{trace}\left(\nabla T_{r}\right)=\sum_{i=1}^{n}\left(\nabla_{E_{i}} T_{r}\right)\left(E_{i}\right)
$$

where $\left\{E_{1}, \ldots, E_{n}\right\}$ is a local orthonormal frame on $M$. Below we will compute $\operatorname{div} T_{r}$, which will be necessary for its later use.

Lemma 3.1. The divergences of the Newton transformations $T_{r}$ are given by the following inductive formula:

$$
\begin{aligned}
& \operatorname{div} T_{0}=0 \\
& \left.\operatorname{div} T_{r}=A\left(\operatorname{div} T_{r-1}\right)+\sum_{i=1}^{n}\left(\bar{R}\left(N, T_{r-1} E_{i}\right) E_{i}\right)^{\mathrm{T}} \cdot\right\}
\end{aligned}
$$

Equivalently, for every tangent field $X \in \mathcal{X}(M)$ it follows that

$$
\left\langle\operatorname{div} T_{r}, X\right\rangle=\sum_{j=1}^{r} \sum_{i=1}^{n}\left\langle\bar{R}\left(N, T_{r-j} E_{i}\right) E_{i}, A^{j-1} X\right\rangle .
$$

A similar expression to (3.4) has recently been obtained by Lima [24] in the Riemannian setting, using a very different argument to ours.

Proof. It is clear that $\operatorname{div} T_{0}=\operatorname{div} I=0$. When $r \geqslant 1$, from the inductive definition of $T_{r}$ we have, for $X, Y \in \mathcal{X}(M)$,

$$
\begin{aligned}
\left(\nabla_{X} T_{r}\right) Y & =\left(\begin{array}{c}
n \\
r
\end{array}\right)\left\langle\nabla H_{r}, X\right\rangle Y+\nabla_{X}\left(A \circ T_{r-1}\right) Y \\
& =\left(\begin{array}{c}
n \\
r
\end{array}\right)\left\langle\nabla H_{r}, X\right\rangle Y+\left(\nabla_{X} A \circ T_{r-1}\right) Y+\left(A \circ \nabla_{X} T_{r-1}\right) Y,
\end{aligned}
$$

so that

$$
\operatorname{div} T_{r}=\sum_{i=1}^{n}\left(\nabla_{E_{i}} T_{r}\right)\left(E_{i}\right)=\left(\begin{array}{l}
n \\
r
\end{array}\right) \nabla H_{r}+\sum_{i=1}^{n}\left(\nabla_{E_{i}} A\right)\left(T_{r-1} E_{i}\right)+A\left(\operatorname{div} T_{r-1}\right) .
$$

Now, using the Codazzi equation (2.5), we get, for $X \in \mathcal{X}(M)$,

$$
\begin{aligned}
\left\langle\left(\nabla_{E_{i}} A\right)\left(T_{r-1} E_{i}\right), X\right\rangle & =\left\langle\left(\nabla_{E_{i}} A\right) X, T_{r-1} E_{i}\right\rangle \\
& =\left\langle\left(\nabla_{X} A\right) E_{i}, T_{r-1} E_{i}\right\rangle+\left\langle\bar{R}\left(X, E_{i}\right) T_{r-1} E_{i}, N\right\rangle \\
& =\left\langle\left(T_{r-1} \circ \nabla_{X} A\right) E_{i}, E_{i}\right\rangle+\left\langle\bar{R}\left(N, T_{r-1} E_{i}\right) E_{i}, X\right\rangle .
\end{aligned}
$$


Therefore,

$$
\begin{aligned}
\left\langle\operatorname{div} T_{r}, X\right\rangle=\left(\begin{array}{l}
n \\
r
\end{array}\right)\left\langle\nabla H_{r},\right. & X\rangle+\operatorname{trace}\left(T_{r-1} \circ \nabla_{X} A\right) \\
& +\left\langle\sum_{i=1}^{n} \bar{R}\left(N, T_{r-1} E_{i}\right) E_{i}, X\right\rangle+\left\langle A\left(\operatorname{div} T_{r-1}\right), X\right\rangle .
\end{aligned}
$$

It remains to show that

$$
\operatorname{trace}\left(T_{r-1} \circ \nabla_{X} A\right)=-\left(\begin{array}{c}
n \\
r
\end{array}\right)\left\langle\nabla H_{r}, X\right\rangle .
$$

We will prove this by computing in a local orthonormal frame on $M$ that diagonalizes $A$. It is worth pointing out that such a frame does not always exist; problems occur when the multiplicity of the principal curvatures changes (also the principal curvatures are not necessarily everywhere differentiable). For that reason, we will work on the subset $M_{0}$ of $M$ consisting of points at which the number of distinct principal curvatures is locally constant. Let us recall that $M_{0}$ is an open dense subset of $M$, and in every connected component of $M_{0}$ the principal curvatures form mutually distinct smooth principal curvature functions and, for such a principal curvature $\kappa$, the assignment $p \mapsto$ $V_{\kappa(p)}(p)$ defines a smooth distribution, where $V_{\kappa(p)}(p) \subset T_{p} M$ denotes the eigenspace associated with $\kappa(p)$ (see, for instance, [8, Paragraph 16.10]). Therefore, for every $p \in M_{0}$ there exists a local orthonormal frame defined on a neighbourhood of $p$ that diagonalizes $A$, that is, $\left\{E_{1}, \ldots, E_{n}\right\}$ such that $A E_{i}=\kappa_{i} E_{i}$, with each $\kappa_{i}$ smooth. In that case,

$$
\left(\nabla_{X} A\right) E_{i}=X\left(\kappa_{i}\right) E_{i}+\sum_{j \neq i}\left(\kappa_{i}-\kappa_{j}\right) \omega_{i}^{j}(X) E_{j},
$$

where, as usual, $\omega_{i}^{j}(X)=\left\langle\nabla_{X} E_{i}, E_{j}\right\rangle$. Then, by (3.1) we have

$$
\begin{aligned}
\operatorname{trace}\left(T_{r-1} \circ \nabla_{X} A\right) & =\sum_{i=1}^{n} \lambda_{i, r-1} X\left(\kappa_{i}\right) \\
& =-\sum_{i=1}^{n} X\left(-\kappa_{i}\right) \sum_{i_{1}<\cdots<i_{r-1}, i_{j} \neq i}\left(-\kappa_{i_{1}}\right) \cdots\left(-\kappa_{i_{r-1}}\right) \\
& =-X\left(\sum_{i_{1}<\cdots<i_{r}}\left(-\kappa_{i_{1}}\right) \cdots\left(-\kappa_{i_{r}}\right)\right)=-\left(\begin{array}{c}
n \\
r
\end{array}\right)\left\langle\nabla H_{r}, X\right\rangle .
\end{aligned}
$$

This proves (3.6) on $M_{0}$ and, by continuity, on $M$. Finally, (3.6) jointly with (3.5) gives (3.3). Equation (3.4) follows easily from (3.3) by an inductive argument.

In particular, when the ambient spacetime $\bar{M}$ has constant sectional curvature, then $(\bar{R}(N, X) Y)^{\mathrm{T}}=0$ for every tangent vector fields $X, Y \in \mathcal{X}(M)$ and equation (3.4) implies that $\operatorname{div} T_{r}=0$ for every $r$.

Corollary 3.2. When the ambient spacetime $\bar{M}$ has constant sectional curvature, the Newton transformations are divergence-free: $\operatorname{div} T_{r}=0$ for each $r$. 


\section{The integral formulae}

In this section we will derive some general integral formulae for compact spacelike hypersurfaces in a conformally stationary spacetime $\bar{M}$. In order to do that, let us consider $K^{\mathrm{T}} \in \mathcal{X}(M)$, the vector field obtained on the hypersurface $M$ by taking the tangential component of $K$, that is,

$$
K^{\mathrm{T}}=K+\langle K, N\rangle N .
$$

Most of the interesting and useful integral formulae in Riemannian geometry are obtained by computing the divergence of certain vector fields and applying the divergence theorem. The interesting integral formulae therefore correspond to vector fields with interesting divergences. Our idea here is to compute the divergence $\operatorname{div}\left(T_{r} K^{\mathrm{T}}\right)$. Using that $\nabla_{X} T_{r}$ is self-adjoint for any $X \in \mathcal{X}(M)$, an easy computation shows that

$$
\operatorname{div}\left(T_{r} K^{\mathrm{T}}\right)=\left\langle\operatorname{div} T_{r}, K^{\mathrm{T}}\right\rangle+\sum_{i=1}^{n}\left\langle\nabla_{E_{i}} K^{\mathrm{T}}, T_{r} E_{i}\right\rangle,
$$

where $\left\{E_{1}, \ldots, E_{n}\right\}$ is a local orthonormal frame on $M$. By taking the covariant derivative in (4.1) and using (2.2) and (2.3), we obtain from (2.1) that

$$
\frac{1}{2}\left(\left\langle\nabla_{X} K^{\mathrm{T}}, Y\right\rangle+\left\langle X, \nabla_{Y} K^{\mathrm{T}}\right\rangle\right)=\phi\langle X, Y\rangle-\langle K, N\rangle\langle A X, Y\rangle,
$$

for tangent vector fields $X, Y \in \mathcal{X}(M)$. Let us choose $\left\{E_{1}, \ldots, E_{n}\right\}$ a local orthonormal frame on $M$ diagonalizing $A$. Then by (3.1) we have

$$
\left\langle\nabla_{E_{i}} K^{\mathrm{T}}, T_{r} E_{i}\right\rangle=\lambda_{i, r}\left\langle\nabla_{E_{i}} K^{\mathrm{T}}, E_{i}\right\rangle=\left\langle E_{i}, \nabla_{T_{r} E_{i}} K^{\mathrm{T}}\right\rangle
$$

and from (4.3) we obtain

$$
\left\langle\nabla_{E_{i}} K^{\mathrm{T}}, T_{r} E_{i}\right\rangle=\phi\left\langle E_{i}, T_{r} E_{i}\right\rangle-\langle K, N\rangle\left\langle A T_{r} E_{i}, E_{i}\right\rangle .
$$

Therefore, using (3.2) and (4.4), equation (4.2) becomes

$$
\begin{aligned}
\operatorname{div}\left(T_{r} K^{\mathrm{T}}\right) & =\left\langle\operatorname{div} T_{r}, K^{\mathrm{T}}\right\rangle+\phi \operatorname{trace}\left(T_{r}\right)-\langle K, N\rangle \operatorname{trace}\left(A \circ T_{r}\right) \\
& =\left\langle\operatorname{div} T_{r}, K^{\mathrm{T}}\right\rangle+c_{r}\left(\phi H_{r}+\langle K, N\rangle H_{r+1}\right),
\end{aligned}
$$

where

$$
c_{r}=(r+1)\left(\begin{array}{c}
n \\
r+1
\end{array}\right) .
$$

Integrating now equation (4.5) on $M$, the divergence theorem implies the following integral formulae.

Theorem 4.1 (Minkowski-type formulae). Let $\psi: M \rightarrow \bar{M}$ be a compact spacelike hypersurface immersed into a conformally stationary spacetime $\bar{M}$. For each $r=1, \ldots, n$ the following formula holds:

$$
\int_{M}\left\langle\operatorname{div} T_{r-1}, K^{\mathrm{T}}\right\rangle \mathrm{d} V+c_{r-1} \int_{M}\left(\phi H_{r-1}+\langle K, N\rangle H_{r}\right) \mathrm{d} V=0,
$$


where

$$
c_{r-1}=r\left(\begin{array}{l}
n \\
r
\end{array}\right)
$$

and $\mathrm{d} V$ is the $n$-dimensional volume element of $M$ with respect to the induced metric and the chosen orientation.

Corollary 4.2. Let $\psi: M \rightarrow \bar{M}$ be a compact spacelike hypersurface immersed into a conformally stationary spacetime $\bar{M}$. Suppose that the following condition holds for some $r$ :

$$
\int_{M}\left\langle\operatorname{div} T_{r-1}, K^{\mathrm{T}}\right\rangle \mathrm{d} V=0
$$

Then

$$
\int_{M}\left(\phi H_{r-1}+\langle K, N\rangle H_{r}\right) \mathrm{d} V=0
$$

We will refer to (4.7) as the $r$ th Minkowski formula for the hypersurface $M$.

Observe that, particularly when the ambient spacetime $\bar{M}$ has constant sectional curvature, we know by Corollary 3.2 that all the Newton transformations are divergence-free and the condition (4.6) automatically holds for every $r$. We then have the following.

Corollary 4.3. Let $\psi: M \rightarrow \bar{M}$ be a compact spacelike hypersurface immersed into a conformally stationary spacetime $\bar{M}$ of constant sectional curvature. Then

$$
\int_{M}\left(\phi H_{r-1}+\langle K, N\rangle H_{r}\right) \mathrm{d} V=0
$$

On the other hand, in the general case we also know that $\operatorname{div} T_{0}=0$, so that the first Minkowski formula always holds in any conformally stationary spacetime. As for the second Minkowski formula, it follows from (3.4) that

$$
\left\langle\operatorname{div} T_{1}, K^{\mathrm{T}}\right\rangle=\sum_{i=1}^{n}\left\langle\bar{R}\left(N, E_{i}\right) E_{i}, K^{\mathrm{T}}\right\rangle=-\overline{\operatorname{Ric}}\left(N, K^{\mathrm{T}}\right) .
$$

Therefore, the second Minkowski formula also holds for those hypersurfaces satisfying that $K^{\mathrm{T}}$ is orthogonal to $\overline{\mathcal{R} i c}(N)$, where $\overline{\mathcal{R}} i c$ is the Ricci operator of $\bar{M}$, that is, $\langle\overline{\mathcal{R} i c}(V), W\rangle=\overline{\operatorname{Ric}}(V, W)$ for every $V, W \in \mathcal{X}(\bar{M})$. In particular, this happens when the ambient spacetime is Einstein.

Our Minkowski-type formulae widely generalize previous integral formulae obtained by the first author together with Romero and Sánchez. Specifically, in [7] they obtained the first and second Minkowski formulae for compact spacelike hypersurfaces in conformally stationary spacetimes, and applied them to the study of compact spacelike hypersurfaces with constant mean curvature (see also $[\mathbf{5}, \mathbf{6}]$ for a first version of those formulae in the case where the ambient spacetime is a generalized Robertson-Walker spacetime). More recently, Montiel [26] has given another proof of the first and second Minkowski formulae in the case where the ambient spacetime $\bar{M}$ is equipped with a conformal timelike vector 
field $K$ which is also closed, in the sense that its metrically equivalent 1-form is closed. Equivalently, that means that

$$
\bar{\nabla}_{V} K=\phi V
$$

for every vector field $V \in \mathcal{X}(\bar{M})$, for a certain smooth function $\phi \in \mathcal{C}^{\infty}(\bar{M})$.

Following the original ideas of Hsiung in his proof of Minkowski formulae for hypersurfaces in Euclidean spaces [20], Montiel's approach uses the method of parallel hypersurfaces, which has a very nice geometric interpretation. However, that method is very difficult to carry out for successive higher-order Minkowski formulae, since it involves covariant derivatives of the Ricci tensor of $\bar{M}$. Although more analytic than geometric, our method has the advantage of working for any higher-order mean curvature.

\section{Umbilicity of constant $r$-mean curvature compact spacelike hypersurfaces}

In this section we will derive some applications of our Minkowski formulae for the case of spacelike hypersurfaces immersed into a conformally stationary spacetime with constant sectional curvature. Observe that ambient spaces with constant sectional curvature are a natural setting for studying constant $r$-mean curvature hypersurfaces, since when the ambient space has constant sectional curvature, then every totally umbilical hypersurface $M$ has constant $r$ th mean curvature $H_{r}$ for every $r$. In fact, if $M$ is totally umbilical, then $A=\lambda I$ for a smooth function $\lambda \in \mathcal{C}^{\infty}(M)$. But the Codazzi equation (2.6) implies that $\lambda$ is constant on $M$, and hence every $H_{r}$ is constant.

First of all, observe that if the mean curvature $H_{1}$ is constant, multiplying by the constant $H_{1}$ the first Minkowski formula we get

$$
\int_{M}\left(\phi H_{1}+\langle K, N\rangle H_{1}^{2}\right) \mathrm{d} V=0 .
$$

The second Minkowski formula gives now

$$
\int_{M}\left(\phi H_{1}+\langle K, N\rangle H_{2}\right) \mathrm{d} V=0,
$$

so that subtracting these two formulae we obtain that

$$
\int_{M}\left(H_{1}^{2}-H_{2}\right)\langle K, N\rangle \mathrm{d} V=0 .
$$

Besides, the Cauchy-Schwarz inequality yields

$$
H_{1}^{2}-H_{2}=\frac{1}{n(n-1)}\left(\sum_{i=1}^{n} \kappa_{i}^{2}-\frac{1}{n}\left(\sum_{i=1}^{n} \kappa_{i}\right)^{2}\right) \geqslant 0,
$$

the equality holding only at umbilical points. Therefore, since $\langle K, N\rangle \leqslant-|K|<0$ we deduce that $H_{1}^{2}-H_{2} \equiv 0$ and the hypersurface must be totally umbilical. This yields the following.

Theorem 5.1. The only compact spacelike hypersurfaces with constant mean curvature in a conformally stationary spacetime of constant sectional curvature are the totally umbilical ones. 
Observe that in the proof of this result we have only used the first and second Minkowski formulae. Since those two Minkowski formulae also hold when the ambient spacetime is assumed to be Einstein, the proof of this result also implies that every compact spacelike hypersurface with constant mean curvature in a conformally stationary Einstein spacetime must be totally umbilical [7, Theorem 3.2]. In particular, every compact spacelike hypersurface with constant mean curvature in an Einstein generalized Robertson-Walker spacetime (necessarily with closed Riemannian fibre) must be totally umbilical [6, Proposition 1].

Since $H_{0}=1$ by definition, Theorem 5.1 can also be read as follows. The only compact spacelike hypersurfaces in a conformally stationary spacetime of constant sectional curvature having $H_{0}$ and $H_{1}$ both constant are the totally umbilical ones. The same argument as above yields the following generalization to the case of any two consecutive $r$ th mean curvatures.

Theorem 5.2. In a conformally stationary spacetime $\bar{M}^{n+1}$ of constant sectional curvature, the only compact spacelike hypersurfaces having $H_{r-1}$ and $H_{r}$ both constant, with $1 \leqslant r \leqslant n-1$, are the totally umbilical ones.

Proof. Multiplying by the constant $H_{r}$ the $r$ th Minkowski formula (4.7) we get

$$
\int_{M}\left(\phi H_{r} H_{r-1}+\langle K, N\rangle H_{r}^{2}\right) \mathrm{d} V=0
$$

and multiplying by the constant $H_{r-1}$ the $(r+1)$ th Minkowski formula we have

$$
\int_{M}\left(\phi H_{r} H_{r-1}+\langle K, N\rangle H_{r-1} H_{r+1}\right) \mathrm{d} V=0 .
$$

Subtracting these two formulae we now obtain that

$$
\int_{M}\left(H_{r}^{2}-H_{r-1} H_{r+1}\right)\langle K, N\rangle \mathrm{d} V=0 .
$$

On the other hand, it is known that the following generalization of the Cauchy-Schwarztype inequality holds true (see, for instance, [18, Theorem 51, p. 52, Theorem 144, p. 104]):

$$
H_{r}^{2}-H_{r-1} H_{r+1} \geqslant 0
$$

the equality holding only at umbilical points. Therefore, since $\langle K, N\rangle \leqslant-|K|<0$, we deduce as above that $H_{r}^{2}-H_{r-1} H_{r+1} \equiv 0$ and the hypersurface must be totally umbilical.

In [26, Theorem 8], Montiel gave a uniqueness result for compact spacelike hypersurfaces with constant scalar curvature immersed into a conformally stationary spacetime with constant sectional curvature which is equipped with a closed conformal timelike vector field. From now on, when the conformal timelike vector field of $\bar{M}$ is closed, we will say that $\bar{M}$ is a conformally stationary-closed spacetime. On the other hand, recall 
that when the ambient spacetime has constant sectional curvature $\bar{c}$, then the scalar curvature of the hypersurface $S$ is related to $H_{2}$ by $S=n(n-1)\left(\bar{c}-H_{2}\right)$. Below we extend Montiel's result to the case of conformally stationary spacetimes, not necessarily with a closed conformal timelike field.

Theorem 5.3. Let $M^{n}, n \geqslant 3$, be a compact spacelike hypersurface immersed into a conformally stationary spacetime $\bar{M}$ of constant sectional curvature $\bar{c}$. If $M$ has constant scalar curvature $S$ such that $S<n(n-1) \bar{c}$ (equivalently, $H_{2}$ is a positive constant), then $M$ is totally umbilical in $\bar{M}$.

Proof. Multiplying by the constant $H_{2}$ the first Minkowski formula and subtracting the third Minkowski formula, we obtain

$$
\int_{M}\left(H_{1} H_{2}-H_{3}\right)\langle K, N\rangle \mathrm{d} V=0 .
$$

Since $H_{2}>0$, we know from (5.1) that $H_{1}^{2} \geqslant H_{2}>0$, and $H_{1}$ does not vanish on $M$. By choosing the appropriate orientation, we may suppose that $H_{1}>0$ on $M$. This can be done because $\mathrm{H}_{2}$ does not depend on the chosen orientation (in fact, $\mathrm{H}_{2}$ is an intrinsic quantity). Besides, we also know by (5.2) that $H_{2}^{2}-H_{1} H_{3} \geqslant 0$, which implies

$$
H_{1} H_{2}-H_{3} \geqslant H_{1} H_{2}-\frac{H_{2}^{2}}{H_{1}}=\frac{H_{2}}{H_{1}}\left(H_{1}^{2}-H_{2}\right) \geqslant 0,
$$

with equality at the umbilical points. Therefore, from the integral formula (5.3) we deduce that $H_{1} H_{2}-H_{3} \equiv 0$ and $M$ is totally umbilical.

It is worth pointing out that for surfaces in de Sitter 3 -space $\mathbb{S}_{1}^{3}$, the hypothesis $S<$ $n(n-1) \bar{c}$ in Theorem 5.3 is unnecessary [24, Theorem 4.4]. However, when $n \geqslant 3$ it is still unknown whether that hypothesis can be removed for hypersurfaces in $\mathbb{S}_{1}^{n+1}$ (see, for instance, $[\mathbf{2}, \mathbf{1 1}, \mathbf{2 3}, \mathbf{3 9}, \mathbf{4 0}])$.

From now on, we will restrict ourselves to the case of conformally stationary-closed spacetimes. Observe that this includes the case of generalized Robertson-Walker spacetimes. Besides, observe that from (2.1), the function $\phi$ can be characterized as

$$
\phi=\frac{1}{n+1} \operatorname{Div} K
$$

where 'Div' denotes the divergence of $\bar{M}$. The following results will be essential in the rest of our applications.

Lemma 5.4 (existence of an elliptic point). Let $\bar{M}$ be a conformally stationaryclosed spacetime (with closed conformal field $K$ ), and let $M$ be a (not necessarily compact) spacelike hypersurface immersed into $\bar{M}$. Assume that Div $K$ does not vanish at a point of $M$ where the restriction of $|K|$ to $M,|K|_{M}=\left.\sqrt{-\langle K, K\rangle}\right|_{M}$, attains a local minimum. Then there exists an elliptic point $p_{0} \in M$. 
Corollary 5.5. Let $\bar{M}$ be a conformally stationary-closed spacetime (with closed conformal field $K$ ), and let $M$ be a compact spacelike hypersurface immersed into $\bar{M}$. If Div $K$ does not vanish on $M$, then there exists an elliptic point $p_{0} \in M$.

Here, by an elliptic point we mean a point where all the principal curvatures $\kappa_{i}\left(p_{0}\right)$ have the same sign. The local condition that Div $K$ does not vanish at a point where $|K|_{M}$ attains a local minimum cannot be dropped, as shown in the following example.

Example 5.6. Let $\bar{M}=-I \times_{f} F^{n}$ be a generalized Robertson-Walker spacetime with compact Riemannian fibre $F$ and warping function $f$. In that case, $K(t, p)=$ $f(t)(\partial / \partial t)_{(t, p)}$ and $\operatorname{Div} K=(n+1) \phi(t, p)=(n+1) f^{\prime}(t)$. Then for each $t_{0} \in I$, the spacelike slice

$$
M_{t_{0}}=\left\{t_{0}\right\} \times F
$$

is a compact totally umbilical hypersurface with constant $r$-mean curvature $H_{r}\left(t_{0}\right)=$ $\left(f^{\prime}\left(t_{0}\right) / f\left(t_{0}\right)\right)^{r}$ (for the details, see [5]). In particular, if $f^{\prime}\left(t_{0}\right)=0$, then $M_{t_{0}}$ is a compact totally geodesic hypersurface without elliptic points.

Proof of Lemma 5.4. Assume that there exists a point $p_{\min } \in M$ where the positive function $|K|_{M}$, or equivalently the function $u=-\left.\langle K, K\rangle\right|_{M}$, attains a local minimum, with $\operatorname{Div} K\left(p_{\text {min }}\right) \neq 0$ (or equivalently $\phi\left(p_{\text {min }}\right) \neq 0$ ). Therefore,

$$
\nabla u\left(p_{\min }\right)=0 \text { and } \nabla^{2} u_{p_{\min }}(v, v) \geqslant 0
$$

for every $v \in T_{p_{\min }} M$. Using that $\bar{\nabla}_{X} K=\phi X$ for every vector field $X$, an easy computation shows that the gradient of $u$ is given by

$$
\nabla u=-2 \phi K^{\mathrm{T}} .
$$

As for its Hessian, we have for every tangent vector field $X \in \mathcal{X}(M)$ that

$$
\begin{aligned}
\nabla^{2} u(X, X) & =\left\langle\nabla_{X}(\nabla u), X\right\rangle=-2 X(\phi)\left\langle X, K^{\mathrm{T}}\right\rangle-2 \phi\left\langle\nabla_{X} K^{\mathrm{T}}, X\right\rangle \\
& =-2 X(\phi)\left\langle X, K^{\mathrm{T}}\right\rangle-2 \phi^{2}|X|^{2}+2 \phi\langle K, N\rangle\langle A X, X\rangle,
\end{aligned}
$$

since $\left\langle\nabla_{X} K^{\mathrm{T}}, X\right\rangle=\phi\langle X, X\rangle-\langle K, N\rangle\langle A X, X\rangle$ from (4.3). Therefore, at the point $p_{\min }$ we get

$$
K^{\mathrm{T}}\left(p_{\min }\right)=0, \quad\langle K, N\rangle\left(p_{\min }\right)=-\sqrt{u\left(p_{\min }\right)},
$$

and

$$
\frac{1}{2} \nabla^{2} u_{p_{\min }}(v, v)=-\phi^{2}\left(p_{\min }\right)|v|^{2}-\phi\left(p_{\min }\right) \sqrt{u\left(p_{\min }\right)}\left\langle A_{p_{\min }} v, v\right\rangle \geqslant 0
$$

for every $v \in T_{p_{\min }} M$. Let us assume, for instance, that $\operatorname{Div} K\left(p_{\min }\right)$ (or equivalently $\phi\left(p_{\min }\right)$ ) is positive (the proof for the case where $\operatorname{Div} K\left(p_{\min }\right)$ is negative is similar but there is an essential difference in the sign of the principal curvatures, as can be seen in Remark 5.7 below). Choosing now $\left\{e_{1}, \ldots, e_{n}\right\}$ a basis of principal directions at $p_{\min }$, we conclude from (5.4) that

$$
\kappa_{i}\left(p_{\min }\right) \leqslant \frac{-\phi\left(p_{\min }\right)}{\sqrt{u\left(p_{\min }\right)}}<0, \quad i=1, \ldots, n
$$


Remark 5.7. It is worth pointing out that if $\operatorname{Div} K\left(p_{\min }\right)$ is negative, then the elliptic point, which is also the point $p_{\min }$ where $u$ attains its minimum, now satisfies

$$
\kappa_{i}\left(p_{\min }\right) \geqslant \frac{-\phi\left(p_{\min }\right)}{\sqrt{u\left(p_{\min }\right)}}>0, \quad i=1, \ldots, n .
$$

This makes sense since we are orienting $M$ by the timelike unit normal field $N$ which is the same time-orientation as $K$.

Remark 5.8. The hypothesis on Div $K$ has both a geometric and a physical content. Actually, from a mathematical point of view the fact that Div $K$ never vanishes on $M$ prevents the hypersurface from having critical points of the metric $\langle$,$\rangle along the integral$ curves of $K$ as

$$
\mathcal{L}_{K}\langle,\rangle=2 \phi\langle,\rangle=\frac{2}{n+1} \operatorname{Div} K\langle,\rangle .
$$

Recall here that for a compact hypersurface in Euclidean space, an elliptic point occurs at a critical point (of maximum or minimum) of the squared length of the position vector, where the position vector is orthogonal to the hypersurface. The same process is used in the proof of Lemma 5.4: we seek a point $p_{\min }$ where the positive function $-\langle K, K\rangle$ attains a minimum on $M$ to have $K\left(p_{\min }\right)$ orthogonal to $M$. However, since now $\nabla\langle K, K\rangle=2 \phi K^{\mathrm{T}}$, to guarantee that $K\left(p_{\min }\right)$ is orthogonal to $M$ we need to have $\phi\left(p_{\min }\right) \neq 0$. In the Euclidean case, we do not need any additional hypothesis on the divergence of the position vector because in that case it is homothetic with constant $\phi \equiv 1 \neq 0$.

On the other hand, from a physical point of view, the normalized vector field

$$
\mathcal{K}=\frac{1}{\sqrt{-\langle K, K\rangle}} K
$$

is a reference frame, that is, a vector field each of whose integral curves is an observer $[\mathbf{3 5}$, Definition 2.3.1]. Using the fact that $K$ is conformal, it easily follows that

$$
\operatorname{Div} \mathcal{K}=\frac{n \phi}{\sqrt{-\langle K, K\rangle}} .
$$

Therefore, if $\phi>0$ on a subset of $\bar{M}$, then $\operatorname{Div} \mathcal{K}>0$ on that subset, which indicates that the observers in $\mathcal{K}$ are on average spreading apart. Similarly, $\phi<0$ on a subset of $\bar{M}$ indicates that the observers come together [35, p. 121].

For the case of generalized Robertson-Walker spacetimes, Corollary 5.5 reads as follows.

Corollary 5.9. Let $\bar{M}=-I \times_{f} F$ be a generalized Robertson-Walker spacetime with (necessarily compact) Riemannian fibre $F$ and warping function $f$, and let $M$ be a compact spacelike hypersurface immersed into $\bar{M}$. Let $t_{\min }$ and $t_{\max }$ denote, respectively, the minimum and the maximum values of $\left.\pi\right|_{M}$, where $\pi=\pi_{I}:-I \times_{f} F \rightarrow I$ is the projection function on $I$. If $f^{\prime}(t) \neq 0$ for every $t_{\min } \leqslant t \leqslant t_{\max }$, then $M$ has an elliptic point. 
In other words, every compact spacelike hypersurface in $-I \times_{f} F$ which is contained in a timelike bounded region

$$
\Omega\left(t_{1}, t_{2}\right)=\left(t_{1}, t_{2}\right) \times F=\left\{(t, p) \in \bar{M}: t_{1}<t<t_{2}\right\}
$$

with $f^{\prime}(t) \neq 0$ for all $t \in\left(t_{1}, t_{2}\right)$, has an elliptic point.

As an application of Corollary 5.5, we are able to extend Theorems 5.1 and 5.3 to the case of higher-order mean curvatures in the following terms. Before stating the result, let us recall that if $\bar{M}$ is a conformally stationary-closed spacetime with closed conformal field $K$, then $K$ determines a foliation $\mathcal{F}(K)$ of $\bar{M}$ by totally umbilical spacelike hypersurfaces with constant mean curvature (and hence, constant $r$-mean curvature) [26, Proposition 1]. In [26, Theorem 5] Montiel gave a classification of the compact umbilical hypersurfaces with constant mean curvature in such ambient spacetimes in terms of that foliation.

Theorem 5.10. Let $M^{n}, n \geqslant 3$, be a compact spacelike hypersurface immersed into a conformally stationary-closed spacetime $\bar{M}$ of constant sectional curvature $\bar{c}$, with a closed conformal timelike vector field $K$ on $\bar{M}$. Assume that Div $K$ does not vanish on $M$. If $M$ has constant $r$ th mean curvature $H_{r}, 1 \leqslant r \leqslant n$, then $M$ is totally umbilical in $\bar{M}$. Besides we have the following classification.

(i) If $\bar{c} \leqslant 0$, then $M$ is a leaf of the foliation $\mathcal{F}(K)$.

(ii) If $\bar{c}>0$, then $M$ is either a leaf of the foliation $\mathcal{F}(K)$ or a round umbilical sphere in a locally de Sitter space.

Proof. When $r=1$, the umbilicity of $M$ was previously proved in Theorem 5.1 without the hypothesis of $K$ being closed and Div $K \neq 0$. For the case $r \geqslant 2$, let us assume, for instance, that Div $K$ is positive on $M$. Then we know from Corollary 5.5 that there exists a point $p_{0} \in M$ where all the principal curvatures are negative, $\kappa_{i}\left(p_{0}\right)<0$. Now, following the ideas of Montiel and Ros in [27, Lemma 1] and their use of Gärding inequalities [16] (taking into account our sign convention in the definition of $H_{j}$ ), it follows that the constant $H_{r}=H_{r}\left(p_{0}\right)$ is positive and

$$
H_{r-1}(p) \geqslant H_{r}^{(r-1) / r}>0
$$

and

$$
H_{1}(p) \geqslant H_{r}^{1 / r}>0
$$

at each point $p \in M$. Moreover, the equality in the above inequalities happens only at umbilical points.

Therefore, since $\phi>0$ on $M$,

$$
\phi H_{r-1} \geqslant \phi H_{r}^{(r-1) / r}
$$


Integrating this inequality, and using the first and the $r$ th Minkowski formulae, we obtain

$$
\begin{aligned}
H_{r} \int_{M}\langle K, N\rangle \mathrm{d} V & =-\int_{M} \phi H_{r-1} \mathrm{~d} V \leqslant-H_{r}^{(r-1) / r} \int_{M} \phi \mathrm{d} V \\
& =H_{r}^{(r-1) / r} \int_{M}\langle K, N\rangle H_{1} \mathrm{~d} V
\end{aligned}
$$

that is,

$$
\int_{M}\left(H_{1}-H_{r}^{1 / r}\right)\langle K, N\rangle \mathrm{d} V \geqslant 0,
$$

with equality if and only if $M$ is totally umbilical. Using now (5.5) we conclude that $H_{1}-H_{r}^{1 / r} \equiv 0$, and hence $M$ is totally umbilical.

Finally, applying the above classification of the compact umbilical spacelike hypersurfaces given by Montiel, we conclude the result. Observe that the fact that Div $K$ does not vanish on $M$ prevents $M$ from being totally geodesic, ruling out the last alternative of Montiel's classification, finishing the proof.

Remark 5.11. When $r \geqslant 2$, it is natural to ask oneself if the hypothesis on Div $K$ in Theorem 5.10 is absolutely necessary in order to conclude the umbilicity of the hypersurface. Answering this question would mean finding an example of a non-umbilic compact spacelike hypersurface with constant $H_{r}$ (for which Div $K$ necessarily vanishes at some point). This question remains open even in the simplest case of spacelike hypersurfaces in de Sitter space. Observe that in the case where $\bar{M}=\mathbb{S}_{1}^{n+1} \subset \mathbb{L}^{n+2}$ is de Sitter space, for every fixed timelike unit vector $a \in \mathbb{L}^{n+2},\langle a, a\rangle=-1$, the vector field $K(x)=a-\langle a, x\rangle x$ defines a closed conformal timelike vector field on $\mathbb{S}_{1}^{n+1}$ with $\phi(x)=-\langle a, x\rangle$. Therefore, by Theorem 5.10 if $M$ were a non-umbilic compact spacelike hypersurface in de Sitter space with constant $H_{r}$, then for every timelike unit vector $a \in \mathbb{L}^{n+2}, M$ could not be contained in any of the two open de Sitter half-spaces determined by $a,\left\{x \in \mathbb{S}_{1}^{n+1}:\langle x, a\rangle>0\right\}$ and $\left\{x \in \mathbb{S}_{1}^{n+1}:\langle x, a\rangle<0\right\}$. Let us recall that the Euclidean sphere $\mathbb{S}^{n+1}$ does contain non-umbilical compact hypersurfaces with constant $H_{r}$ which are not contained in an open half-sphere, for instance, the tori $\mathbb{S}^{n-m}(\varrho) \times \mathbb{S}^{m}\left(\sqrt{1-\varrho^{2}}\right) \subset \mathbb{S}^{n+1}$, for every $1 \leqslant m \leqslant n-1$ and $0<\varrho<1$. These tori are examples of non-umbilic compact (isoparametric) hypersurfaces in the sphere with constant $H_{r}$, for every $r \geqslant 1$. The situation in de Sitter space is radically different since the compactness of the spacelike hypersurface forces it to be diffeomorphic to an $n$-sphere, which is a strong topological restriction. Indeed, it is our impression that there does not exist any non-umbilic compact spacelike hypersurface in de Sitter space with constant $H_{r}$, although it has not yet been proved.

In particular, for the case of generalized Robertson-Walker spacetimes, using the characterization of their totally umbilical compact spacelike hypersurfaces given by Montiel $[\mathbf{2 6}$, Theorem 6] we can conclude from Theorem 5.10 the following.

Corollary 5.12. Let $\bar{M}=-I \times_{f} F$ be a generalized Robertson-Walker spacetime (necessarily with compact Riemannian fibre $F$ ) with constant sectional curvature. Then, the only compact spacelike hypersurfaces immersed into $\bar{M}$ with constant $r$-mean curvature on which $f^{\prime}$ does not vanish are the non-totally geodesic spacelike slices $\{t\} \times F$, 
with $t \in I$ and $f^{\prime}(t) \neq 0$, unless in the case where $\bar{M}$ is isometric to de Sitter spacetime in a neighbourhood of the hypersurface $M$, which must be a round umbilical hypersphere.

Remark 5.13. It is not difficult to see that an $(n+1)$-dimensional generalized Robertson-Walker spacetime $\bar{M}=-I \times{ }_{f} F^{n}, n \geqslant 2$, has constant sectional curvature $\bar{c}$ if and only if the Riemannian fibre $F$ has constant sectional curvature $c$ and the warping function $f$ satisfies the differential equations

$$
\frac{f^{\prime \prime}}{f}=\bar{c} \quad \text { and } \quad \frac{\left(f^{\prime}\right)^{2}+c}{f^{2}}=\bar{c}
$$

(see, for instance, [8, Corollary 9.107]). The positive solutions to (5.6) are given by the following expressions (in each case, the interval of definition $I$ of $f$ is the maximal one where $f$ is positive):

$$
\begin{array}{llll}
\text { Case 1 } & \bar{c}>0 & c>0 & f(t)=a \mathrm{e}^{\sqrt{\bar{c}} t}+\frac{c}{4 a \bar{c}} \mathrm{e}^{-\sqrt{\bar{c}} t}, \quad a>0, \\
\text { Case 2 } & \bar{c}>0 & c=0 & f(t)=a \mathrm{e}^{\varepsilon \sqrt{\bar{c}} t}, \quad a>0, \quad \varepsilon= \pm 1, \\
\text { Case 3 } & \bar{c}>0 & c<0 & f(t)=a \mathrm{e}^{\sqrt{\bar{c}} t}+\frac{c}{4 a \bar{c}} \mathrm{e}^{-\sqrt{\bar{c}} t}, \quad a \neq 0, \\
\text { Case 4 } & \bar{c}=0 & c=0 & f(t)=a, \quad a>0, \\
\text { Case 5 } & \bar{c}=0 & c<0 & f(t)=\varepsilon \sqrt{-c} t+a, \quad \varepsilon= \pm 1, \\
\text { Case 6 } & \bar{c}<0 & c<0 & f(t)=a_{1} \cos (\sqrt{-\bar{c}} t)+a_{2} \sin (\sqrt{-\bar{c}} t), \quad a_{1}^{2}+a_{2}^{2}=c / \bar{c} .
\end{array}
$$

In particular, in Cases 2, 3 and 5 the derivative of $f$ satisfies $f^{\prime}(t) \neq 0$ for all $t \in I$, so that the requirement that $f^{\prime}$ does not vanish on $M$ always holds. On the other hand, in Case $1, I=\mathbb{R}$ and $f^{\prime}(t)=0$ at the value $t_{*}=\left(\frac{1}{2} \sqrt{\bar{c}}\right) \log \left(c / 4 a^{2} \bar{c}\right)$. Therefore, $f^{\prime}$ does not vanish on $M$ if and only if the image of $M$ under the immersion is contained either in $\left(-\infty, t_{*}\right) \times F$ or in $\left(t_{*}, \infty\right) \times F$.

\section{Hypersurfaces with linearly related $r$-mean curvatures}

When the ambient spacetime is a conformally stationary-closed spacetime with constant sectional curvature, we obtain the following umbilicity result.

Theorem 6.1. Let $M^{n}$ be a compact spacelike hypersurface immersed into a conformally stationary-closed spacetime $\bar{M}$ with constant sectional curvature $\bar{c}$. Assume that Div $K$ does not vanish on $M$, where $K$ denotes the closed conformal timelike vector field on $\bar{M}$. Assume that for integers $r$ and $s$, with either $0 \leqslant r<s<n$ or $0<r<s \leqslant n$, the higher-order mean curvatures are linearly related by

$$
H_{s}=a_{r} H_{r}+\cdots+a_{s-1} H_{s-1}
$$

for non-negative numbers $a_{r}, \ldots, a_{s-1}$. Then $M$ is totally umbilical in $\bar{M}$. Besides we have the following classification. 
(i) If $\bar{c} \leqslant 0$, then $M$ is a leaf of the foliation $\mathcal{F}(K)$.

(ii) If $\bar{c}>0$, then $M$ is either a leaf of the foliation $\mathcal{F}(K)$ or a round umbilical sphere in a locally de Sitter space.

\section{Proof.}

Case 1. Let us assume first that $s<n$. Using the linear relation (6.1) and the $(s+1)$ th Minkowski formula, we obtain

$$
\int_{M}\langle K, N\rangle H_{s+1} \mathrm{~d} V=-\sum_{j=r}^{s-1} a_{j} \int_{M} \phi H_{j} \mathrm{~d} V=\sum_{j=r}^{s-1} a_{j} \int_{M}\langle K, N\rangle H_{j+1} \mathrm{~d} V,
$$

where we have also used the $j$ th Minkowski formulae for $j=r, \ldots, s-1$. That is,

$$
\int_{M}\langle K, N\rangle\left(H_{s+1}-\sum_{j=r}^{s-1} a_{j} H_{j+1}\right) \mathrm{d} V=0 .
$$

We claim that

$$
H_{s+1} \leqslant \sum_{j=r}^{s-1} a_{j} H_{j+1}
$$

with equality at umbilic points. If our claim is true, then we can conclude the result in the same way as in our other previous results.

In order to prove our claim, we may assume without loss of generality that Div $K$ is positive on $M$. Then we know from Corollary 5.5 that there exists a point $p_{0} \in M$ where all the principal curvatures are negative. Denote by $M_{s}$ the connected component of $\left\{p \in M: H_{s}(p)>0\right\}$ containing the elliptic point $p_{0}$ (recall our sign convention for $H_{s}$ ). It is clear that $M_{s}$ is a non-empty open subset of $M$. We will show that it is also closed. In fact, since $H_{s}\left(p_{0}\right)>0$, there exists at least one positive coefficient $a_{j}$, say $a_{l}>0$. By using again Gärding inequalities as in [27, Lemma 1] we know that at each point $p \in M_{s}$

$$
H_{j}^{s / j}(p) \geqslant H_{s}(p)>0, \quad 1 \leqslant j \leqslant s-1 .
$$

In particular, since $a_{j} \geqslant 0$, at each point $p \in M_{s}$ we have

$$
H_{s}(p) \geqslant a_{l} H_{l}(p) .
$$

If $l=0$, then $H_{s} \geqslant a_{0}>0$ on $M_{s}$, proving that $M_{s}$ is closed. If $l \geqslant 1$, then we have on $M_{s}$

$$
H_{l}^{s / l} \geqslant H_{s} \geqslant a_{l} H_{l}>0 .
$$

Hence $H_{l}^{(s-l) / l} \geqslant a_{l}$ on $M_{s}$, which gives

$$
H_{s} \geqslant a_{l} a_{l}^{l /(s-l)}=a_{l}^{s /(s-l)}>0,
$$

showing that also in this case $M_{s}$ is closed. 
Therefore, $M_{s}=M$ and (6.4) holds at every point $p \in M$. In particular, $H_{j}>0$ for every $j, 1 \leqslant j \leqslant s$. Moreover, we also know by $(5.2)$ that

$$
H_{j}^{2}-H_{j-1} H_{j+1} \geqslant 0
$$

with equality at the umbilical points. Since each $H_{j}>0$, for $1 \leqslant j \leqslant s$, this is equivalent to

$$
\frac{H_{s+1}}{H_{s}} \leqslant \frac{H_{s}}{H_{s-1}} \leqslant \cdots \leqslant \frac{H_{j+1}}{H_{j}} \leqslant \cdots \leqslant \frac{H_{2}}{H_{1}} \leqslant H_{1}
$$

with equality at any stage only at umbilical points. Observe that the first inequality in (6.5) holds independently of the sign of $H_{s+1}$. From (6.5), and using (6.1), we obtain that

$$
\frac{H_{s+1}}{H_{s}} \leqslant \frac{H_{s}}{H_{s-1}}=\sum_{j=r}^{s-1} a_{j} \frac{H_{j}}{H_{s-1}} \leqslant \sum_{j=r}^{s-1} a_{j} \frac{H_{j+1}}{H_{s}},
$$

which means that the claim in (6.3) is true, with equality at umbilic points. By (6.2) we then conclude that

$$
H_{s+1}=\sum_{j=r}^{s-1} a_{j} H_{j+1},
$$

which implies that $M$ is totally umbilical. The last statement of the theorem runs as in Theorem 5.10. This finishes the proof in the case that $s<n$.

Case 2. Let us now assume that $s=n$ but $r>0$. In that case, using the linear relation (6.1) and the $n$th Minkowski formula we have that

$$
\int_{M} \phi H_{n-1} \mathrm{~d} V=-\sum_{j=r}^{n-1} a_{j} \int_{M}\langle K, N\rangle H_{j} \mathrm{~d} V=\sum_{j=r}^{n-1} a_{j} \int_{M} \phi H_{j-1} \mathrm{~d} V,
$$

where we have also used the $j$ th Minkowski formulae for $j=r, \ldots, n-1$. Therefore,

$$
\int_{M} \phi\left(H_{n-1}-\sum_{j=r}^{n-1} a_{j} H_{j-1}\right) \mathrm{d} V=0 .
$$

We claim now that

$$
H_{n-1} \geqslant \sum_{j=r}^{n-1} a_{j} H_{j-1},
$$

with equality at umbilic points. If this claim is true, then we can conclude the result as in Case 1.

To prove the claim (6.6), we may assume again without loss of generality that Div $K$ is positive on $M$, so that by Corollary 5.5 there exists a point $p_{0} \in M$ where all the principal curvatures are negative. The same reasoning as in Case 1 now shows that $M_{n}=\left\{p \in M: H_{n}(p)>0\right\}=M$ and $H_{j}^{n / j}(p) \geqslant H_{n}(p)>0$ at each point $p \in M$, for every $1 \leqslant j \leqslant n-1$. By Newton inequalities (5.2) we then have

$$
\frac{H_{n}}{H_{n-1}} \leqslant \frac{H_{n-1}}{H_{n-2}} \leqslant \cdots \leqslant \frac{H_{j}}{H_{j-1}} \leqslant \cdots \leqslant \frac{H_{2}}{H_{1}} \leqslant H_{1}
$$


with equality at any stage only at umbilical points. From here, and using the linear relation $(6.1)$, we get

$$
\frac{H_{n-1}}{H_{n-2}} \geqslant \frac{H_{n}}{H_{n-1}}=\sum_{j=r}^{n-1} a_{j} \frac{H_{j}}{H_{n-1}} \geqslant \sum_{j=r}^{n-1} a_{j} \frac{H_{j-1}}{H_{n-2}},
$$

what means that the claim (6.6) is true, with equality at umbilic points. This finishes the proof when $s=n$.

Remark 6.2. If $a_{j}=0$ except for some $j=k$, the hypothesis (6.1) becomes

$$
H_{s}=a_{k} H_{k} .
$$

In this case it is not necessary to assume that the coefficient $a_{k}$ is non-negative. Actually, since $H_{s}\left(p_{0}\right)>0$ and $H_{k}\left(p_{0}\right)>0$, then $a_{k}$ is necessarily positive. This case was proved in [4] when the ambient space is de Sitter space, under the additional hypothesis that $H_{k}$ does not vanish on $M$.

Remark 6.3. In the Riemannian setting, a theorem analogous to Theorem 6.1 was proved by Stong $[\mathbf{3 8}]$ for hypersurfaces in the Euclidean space under the additional hypothesis that each $H_{j}$ is positive and the support function does not change its sign on $M$. A similar result is also proved in [38] for hypersurfaces embedded in a Riemannian space of constant sectional curvature having a pole.

On the other hand, in [9] Bivens proved that when the ambient space is the Euclidean space, the hyperbolic space, or the open hemisphere, then every immersed compact hypersurface with $H_{r}$ and $H_{r+1}$ both constant must be a geodesic sphere. Related to this, Koh $[\mathbf{2 2}]$ has recently proved the same kind of result under the hypothesis that the ratio $H_{s} / H_{r}$ is constant, $1 \leqslant r<s \leqslant n$.

\section{A further application}

As another application of our integral formulae we have the following consequence, which holds for a conformally stationary spacetime with constant sectional curvature, with the conformal timelike field not necessarily closed.

Proposition 7.1. Let $M^{n}$ be a compact spacelike hypersurface immersed into a conformally stationary spacetime $\bar{M}$ with constant sectional curvature. If $H_{1}>0, \ldots, H_{r}>0$ and $H_{r}$ is constant, with $2 \leqslant r \leqslant n-1$, then $M$ is totally umbilical in $\bar{M}$.

Proof. As in the proof of Theorem 5.10, we have that

$$
\int_{M}\left(H_{1} H_{r}-H_{r+1}\right)\langle K, N\rangle \mathrm{d} V=0
$$

so that our objective is to see that $H_{1} H_{r}-H_{r+1} \geqslant 0$, with equality at umbilical points. For each $j=1, \ldots, r$ we know from (5.2) that

$$
H_{j}^{2}-H_{j-1} H_{j+1} \geqslant 0
$$


with equality at the umbilical points. Since each $H_{j}>0,1 \leqslant j \leqslant r$, this is equivalent to

$$
H_{1} \geqslant \frac{H_{2}}{H_{1}} \geqslant \cdots \geqslant \frac{H_{r}}{H_{r-1}} \geqslant \frac{H_{r+1}}{H_{r}}
$$

with equality at any stage only at umbilical points. But this implies $H_{1} H_{r}-H_{r+1} \geqslant 0$, with equality at the umbilical points, finishing the proof.

Acknowledgements. L.J.A. was partly supported by DGICYT and Fundación Séneca (PRIDTYC), Spain, and by FUNCAP, Brazil. A.G.C. was partly supported by CNPq, Brazil.

This work was finished while L.J.A. was visiting the Departamento de Matemática of the Universidade Federal do Ceará, Fortaleza, Brazil. He thanks that institution and the members of the department for their hospitality.

The authors thank the referee for valuable suggestions which improved the paper.

\section{References}

1. K. Akutagawa, On spacelike hypersurfaces with constant mean curvature in the de Sitter space, Math. Z. 196 (1987), 13-19.

2. J. A. Aledo, L. J. Alías And A. Romero, Integral formulas for compact spacelike hypersurfaces in de Sitter space. Applications to the case of constant higher order mean curvature, J. Geom. Phys. 31 (1999), 195-208.

3. H. Alencar And A. G. Colares, Integral formulas for the $r$-mean curvature linearized operator of a hypersurface, Ann. Global Analysis Geom. 16 (1998), 203-220.

4. L. J. AlíAS And S.-E. KoH, Remarks on compact spacelike hypersurfaces in de Sitter space with constant higher order mean curvature, J. Geom. Phys. 39 (2001), 46-50.

5. L. J. Alías, A. Romero and M. SÁnchez, Uniqueness of complete spacelike hypersurfaces of constant mean curvature in Generalized Robertson-Walker spacetimes, Gen. Relat. Grav. 27 (1995), 71-84.

6. L. J. Alías, A. Romero ANd M. SÁnchez, Spacelike hypersurfaces of constant mean curvature and Calabi-Bernstein type problems, Tôhoku Math. J. 49 (1997), 337-345.

7. L. J. Alías, A. Romero And M. SÁnchez, Spacelike hypersurfaces of constant mean curvature in certain spacetimes, Nonlin. Analysis 30 (1997), 655-661.

8. A. L. Besse, Einstein manifolds (Springer, 1987).

9. I. Bivens, Integral formulas and hyperspheres in a simply connected space form, Proc. Am. Math. Soc. 88 (1983), 113-118.

10. A. Brasil JR and A. G. Colares, On constant mean curvature spacelike hypersurfaces in Lorentzian manifolds Matemática Contemporânea 17 (1999), 99-136.

11. Q.-M. Cheng And S. IshiKaWA, Spacelike hypersurfaces with constant scalar curvature, Manuscr. Math. 95 (1998), 499-505.

12. S. Y. Cheng And S. T. YAU, Maximal spacelike hypersurfaces in the Lorentz-Minkowski spaces, Ann. Math. 104 (1976), 407-419.

13. A. A. Coley And B. O. J. Tupper, Special conformal Killing vector spacetimes and symmetry inheritance, J. Math. Phys. 30 (1989), 2616-2625.

14. A. A. Coley and B. O. J. Tupper, Fluid spacetimes admitting covariantly constant vectors and tensors, Gen. Relat. Grav. 23 (1991), 1113-1142.

15. D. Earley, J. Isenberg, J. Marsden and V. Moncrief, Homothetic and conformal symmetries of solutions to Einstein's equations, Commun. Math. Phys. 106 (1986), 137158. 
16. L. GÁrding, An inequality for hyperbolic polynomials, J. Math. Mech. 8 (1959), 957-965.

17. A. J. GodDARD, Some remarks on the existence of spacelike hypersurfaces of constant mean curvature, Math. Proc. Camb. Phil. Soc. 82 (1977), 489-495.

18. G. Hardy, J. E. Littlewood and G. Póyla, Inequalities, 2nd edn (Cambridge Mathematical Library, 1989).

19. S. HARRIS, Conformally stationary spacetimes, Class. Quant. Grav. 9 (1992), 1823-1827.

20. C. C. Hsiung, Some integral formulas for closed hypersurfaces, Math. Scand. 2 (1954), 286-294.

21. Y. Kamishima, Completeness of Lorentz manifolds of constant curvature admitting Killing vectors fields, J. Diff. Geom. 37 (1993), 569-601.

22. S.-E. KоH, Sphere theorems by means of the ratio of mean curvature functions, Glasgow Math. J. 42 (2000), 91-95.

23. H. LI, Global rigidity theorems of hypersurfaces, Ark. Mat. 35 (1997), 327-351.

24. B. P. Lima, O Princípio de Omori-Yau e Aplicações. Tese de Doutorado, Universidade Federal do Ceará, Brasil (2000).

25. S. Montiel, An integral inequality for compact spacelike hypersurfaces in de Sitter space and applications to the case of constant mean curvature, Indiana Univ. Math. J. 37 (1988), 909-917.

26. S. Montiel, Uniqueness of spacelike hypersurfaces of constant mean curvature in foliated spacetimes, Math. Ann. 314 (1999), 529-553.

27. S. Montiel AND A. Ros, Compact hypersurfaces: the Alexandrov theorem for higher order mean curvatures, in Differential geometry (ed. B. Lawson and K. Tenenblat), pp. 279-296 (Longman, 1991).

28. B. O'NeILL, Semi-Riemannian geometry with applications to relativity (Academic Press, 1983).

29. J. Ramanathan, Complete spacelike hypersurfaces of constant mean curvature in de Sitter space, Indiana Univ. Math. J. 36 (1987), 349-359.

30. R. C. REILly, Variational properties of functions of the mean curvature for hypersurfaces in space forms, J. Diff. Geom. 8 (1973), 465-477.

31. A. Romero And M. SÁnchez, Completeness of compact Lorentz manifolds admitting a timelike conformal Killing vector field, Proc. Am. Math. Soc. 123 (1995), 2831-2833.

32. A. Romero And M. SÁnchez, An integral inequality on compact Lorentz manifolds and its applications, Bull. Lond. Math. Soc. 28 (1996), 509-513.

33. A. Romero AND M. SÁnCHEz, Bochner's technique on Lorentzian manifolds and infinitesimal conformal symmetries, Pac. J. Math. 186 (1998), 141-148.

34. H. Rosenberg, Hypersurfaces of constant curvature in space forms, Bull. Sci. Math. 117 (1993), 211-239.

35. R. K. SACHS And H. Wu, General relativity for mathematicians, Graduate Texts in Mathematics (Springer, 1977).

36. M. SÁNCHEz, Lorentzian manifolds admitting a Killing vector field, Nonlin. Analysis 30 (1997), 643-654.

37. M. SÁnchez, Structure of Lorentzian tori with a Killing vector field, Trans. Am. Math. Soc. 349 (1997), 1063-1080.

38. R. E. Stong, Some characterizations of Riemann n-spheres, Proc. Am. Math. Soc. 11 (1960), 945-951.

39. Y. Zheng, On spacelike hypersurfaces in the de Sitter space, Ann. Global Analysis Geom. 13 (1995), 317-321.

40. Y. ZHENG, Spacelike hypersurfaces with constant scalar curvature in the de Sitter spaces, Diff. Geom. Applic. 6 (1996), 51-54. 